Check for updates

Cite this: RSC Adv., 2018, 8, 28546

\title{
Incorporation of simvastatin in PLLA membranes for guided bone regeneration: effect of thermal treatment on simvastatin release $\uparrow$
}

\author{
Antonio G. B. Castro, ${ }^{a}$ Dennis W. P. M. Löwik, ${ }^{c}$ Mies J. van Steenbergen, ${ }^{b}$ \\ John A. Jansen, ${ }^{a}$ Jeroen J. J. P. van den Beucken ${ }^{a}$ and Fang Yang (iD *a
}

\begin{abstract}
Electrospun membranes based on biodegradable polymers are promising materials to be used for guided bone regeneration (GBR) therapy. The incorporation of osteostimulatory compounds can improve the biofunctionality of those membranes, making them active players in bone regeneration. Simvastatin has been shown to promote osteogenic differentiation both in vitro and in vivo. However, in most of these systems, the drug was quickly released, not matching the pace of bone regeneration. The aim of this study was to develop poly(L-lactic acid) (PLLA) membranes containing simvastatin (SV) that have a prolonged drug release rate, compatible with GBR applications. To this end, SV was mixed with PLLA and electrospun. The membranes were subjected to a thermal treatment in order to increase the crystallinity of PLLA. Morphological, structural and chemical properties of the electrospun membranes were characterized. The effect of the thermal treatment on the release profile of SV was evaluated by near physiological release experiments at $37^{\circ} \mathrm{C}$. The osteostimulatory potential was determined by in vitro culture of the membranes with rat bone marrow stromal cells (rBMSCs). The results confirmed that the thermal treatment led to an increase in polymer crystallinity and a more sustained release of SV. In vitro assays demonstrate cellular proliferation over time for all the membranes and a significant increase in osteogenic differentiation for the membranes containing SV subjected to

thermal treatment.
\end{abstract}

Received 23rd May 2018

Accepted 5th August 2018

DOI: $10.1039 / \mathrm{c} 8 \mathrm{ra04397c}$

rsc.li/rsc-advances

\section{Introduction}

Dental implants are a common treatment option for replacing missing teeth due to diseases, like periodontitis, or trauma. Successful implant placement requires adequate alveolar ridge dimensions, which are essential to house the implant and provide esthetics and function. ${ }^{1}$ Unfortunately, this is usually not the case due to bone resorption or damage. Therefore, a ridge augmentation technique is often required to regenerate enough bone for successful implant placement. Guided bone regeneration (GBR) is an established strategy for this purpose and based on the creation of a secluded space, in which pluripotent and osteogenic cells are allowed to proliferate and form new bone, whilst soft tissue formation (by e.g. fibroblasts

\footnotetext{
${ }^{a}$ Department of Biomaterials, Radboudumc, Philips van Leydenlaan 25, Nijmegen, 6525 EX, The Netherlands. E-mail:fang.yang@radboudumc.nl

${ }^{b}$ Utrecht Institute for Pharmaceutical Sciences (UIPS), Utrecht University, 3584 CG Utrecht, The Netherlands

'Bio-organic Chemistry, Institute for Molecules and Materials, Radboud University Nijmegen, Heyendaalseweg 135, Nijmegen, 6525 AJ, The Netherlands

† Electronic supplementary information (ESI) available. See DOI: $10.1039 / \mathrm{c} 8 \mathrm{ra04397c}$
}

and epithelial cells) is inhibited. ${ }^{1}$ This can be achieved by a physical barrier, mainly in the form of a membrane. ${ }^{2,3}$

Electrospinning is an optimal technique to create polymeric membranes. Via electrospinning, polymer solutions are ejected under electrical force; the fluid jet is elongated and solidifies to ultrafine fibers before reaching a collector; those randomly deposited ultrafine fibers form non-woven membranes. ${ }^{4}$ The electrospun membranes have a high porosity and surface area-tovolume ratio, resembling the extracellular matrix (ECM) structure. More importantly, the pore size of the electrospun membranes, in general, is less than the average cell size and previous studies have shown that such small pores do not allow cell penetration. ${ }^{5}$

GBR membranes do not possess an active role in local bone regeneration, and specifically synthetic membranes are inherently hydrophobic ${ }^{6,7}$ and biologically inert. ${ }^{8}$ Different strategies can be adopted to overcome these drawbacks. The development of new materials based on polymeric composites $^{5,9}$ or graphene/graphene oxide composites ${ }^{\mathbf{1 0 , 1 1}}$ are promising approaches, leading to the proliferation and osteogenic differentiation of osteoblastic cells. An alternative strategy is the incorporation of osteostimulatory compounds. The incorporation of growth factors, such as bone morphogenetic proteins (BMPs) is a first choice due to their potent osteoinductive effect. ${ }^{12,13}$ However, their incorporation is limited by 
their inactivity when in contact with organic solvents or at physiological conditions, uncontrolled release which can lead to supraphysiologic concentrations and high cost. ${ }^{\mathbf{1 4 , 1 5}}$ Small osteogenic pharmaceutical drugs have shown to be a promising alternative, showing higher chemical stability and a lower cost. ${ }^{16}$ Simvastatin (SV) is used to treat hypercholesterolemia and hyperlipidemia. ${ }^{\mathbf{1 7 , 1 8}}$ Recently, it has also been reported that SV directly promotes osteoblastic differentiation and improves new bone formation by increasing the expression of bone morphogenetic protein-2 (BMP-2), alkaline phosphatase (ALP), osteocalcin and collagen type 1 (COL1) mRNAs in osteoblast-like cells and stem cells. The mechanism associated with the influence of simvastatin on the osteogenic differentiation is not fully understood. However, recent studies point out to a direct action of simvastatin in the activation of the Wnt/ $\beta$-catenin signaling pathway, which is involved in the differentiation of mesenchymal stem cells (MSCs) and the proliferation, differentiation, mineralization and apoptosis of osteoblastic cells. ${ }^{19}$ Promotion of angiogenesis by SV has also been reported. ${ }^{20} \mathrm{SV}$ has been incorporated in different materials, including calcium-phosphate particles and granules, ${ }^{20,21}$ metallic substrates ${ }^{22,23}$ and polymeric particles $^{24}$ micelles $^{25}$ and scaffolds. ${ }^{26,27}$ Electrospun polymeric membranes containing SV have been previously developed, ${ }^{28,29}$ but SV release from these membranes occurred as fast as within 8 days, ${ }^{20,21,24,26}$ which does not match the pace of bone regeneration $\left(\sim 12\right.$ weeks). ${ }^{30}$ Consequently, the need for a system achieving prolonged SV release is apparent.

Thermal treatments such as annealing, ${ }^{31}$ quenching, ${ }^{32}$ or aging $^{33}$ are commonly used to modify the physicochemical properties of polymers. By heating a polymer to a temperature slightly above its cold crystallization temperature $\left(T_{\mathrm{cc}}\right)$, it is possible to increase its overall crystallinity. Here, our goal was to develop an electrospun poly(L-lactic acid) (PLLA) membrane for prolonged SV release. PLLA was chosen due to its biocompatibility, biodegradability, and appropriate mechanical and physicochemical properties. More specifically, PLLA showed no obvious degradation within 3 months, which ensures the mechanical stability of the GBR membranes during the treatment period. ${ }^{34,35}$ PLLA is a semi-crystalline polymer and it has been reported that the change of crystallinity of a polymeric drug carrier significantly affects the release of drugs. ${ }^{36-40}$ Consequently, we utilized thermal treatment of the electrospun PLLA membranes to increase its crystallinity and control the drug release. The morphological properties of the membranes were evaluated by scanning electron microscopy (SEM), physicochemical properties were analyzed by attenuated total reflectance-infrared spectroscopy (ATR-IR), X-ray diffraction (XRD) and differential scanning calorimetry (DSC). The release profile of simvastatin from the membranes with or without thermal treatment was evaluated by in vitro incubation in nearphysiological conditions at $37{ }^{\circ} \mathrm{C}$. The cellular osteogenic response to the membranes and biological activity of SV was determined by in vitro culture of the different membranes with rat bone marrow stromal-cells (rBMSCs) and evaluating cell morphology, proliferation, and osteogenic differentiation over culture time.

\section{Materials and methods}

\section{Preparation of polymeric solutions and fabrication of electrospun membranes}

Poly(L-lactic acid) (PLLA; Purasorb PL65®; Corbion, The Netherlands) solutions were prepared by dissolving the polymer in 1,1,1,3,3,3-hexafluoroisopropanol (HFIP; Fluorochem, UK) to a concentration of $3 \% \mathrm{w} / \mathrm{v}$. Solutions containing simvastatin (SV; Sigma-Aldrich, USA) were prepared by dissolving the SV ( $5 \% \mathrm{w} / \mathrm{w}$ to PLLA) and PLLA in HFIP and left to be stirred overnight. Membranes were prepared by electrospinning (Advanced Surface Technology BV, The Netherlands) and the following parameters were applied: needle diameter $=1.2 \mathrm{~mm}$, feeding rate $=2.0 \mathrm{ml} \mathrm{h}^{-1}$; distance between the needle and the collector $=20 \mathrm{~cm}$; voltage $=19.25 \mathrm{kV}$.

Thermal treatment of the membranes was performed in an oven at $80^{\circ} \mathrm{C}$ for 16 hours.

Four different membranes were produced: pure PLLA membranes (PLLA); PLLA membranes subjected to the thermal treatment (PLLA HT); PLLA membranes containing simvastatin (PLLA + SV); PLLA membranes containing simvastatin and subjected to the thermal treatment (PLLA + SV HT).

\section{Morphological and structural evaluation}

The morphology of the membranes was observed using scanning electron microscopy (SEM; SIGMA 300; Zeiss, The Netherlands). Samples were fixed into aluminum stubs with carbon tape and sputter coated with chromium (thickness of $20 \mathrm{~nm}$ ).

The chemical profile of the membranes was assessed by attenuated total reflectance-infrared spectroscopy (ATR-IR; UATR two; PerkinElmer, The Netherlands) with a resolution of $4.0 \mathrm{~cm}^{-1}$ and a scanning range from $400 \mathrm{~cm}^{-1}$ to $4000 \mathrm{~cm}^{-1}$.

X-ray diffraction (XRD; $\mathrm{X}^{\prime}$ pert $^{3}$ powder; PANalytical, The Netherlands) was performed to determine the crystallographic profile of the membranes. Electrospun membranes as a thin planar layer were placed on a glass holder and scanned. XRD spectra were registered at $40 \mathrm{kV}, 30 \mathrm{~mA}(\mathrm{Cu}-\mathrm{K} \alpha$ radiation with a wavelength of $1.54 \AA$ ) and a $2 \theta$ between 10 and $40^{\circ}$, at a step size of $0.005^{\circ}$.

Crystallinity was determined by modulated differential scanning calorimetry (DSC; Discovery®; TA instruments, The Netherlands). For DSC analysis, samples were placed in perforated aluminum pans, to avoid overpressure, and scanned from 20 to $200{ }^{\circ} \mathrm{C}$ at a heating rate of $2{ }^{\circ} \mathrm{C} \mathrm{min}^{-1}$. The degree of crystallinity $\left(\chi_{\mathrm{c}} \%\right)$ was calculated considering the ratio between the experimental heat of melting $\left(\Delta H_{\mathrm{m}}\right)$ minus the cold crystallization heat $\left(\Delta H_{\mathrm{cc}}\right)$ and the heat of melting of $100 \%$ crystalline PLLA $\left(\Delta H_{\mathrm{m}}^{\circ}=93.6 \mathrm{~J} \mathrm{~g}^{-1}\right),{ }^{41}$ according to the following equation:

$$
\chi_{\mathrm{c}} \%=\frac{\left[\Delta H_{\mathrm{m}}-\Delta H_{\mathrm{cc}}\right]}{\Delta H_{\mathrm{m}}^{\circ}}
$$

\section{Release of simvastatin (SV) in an aqueous medium}

Membranes containing SV with or without thermal treatment $\left(\approx 0.8 \mathrm{mg}\right.$ each sample) were immersed in $1 \mathrm{ml}$ of PBS at $37^{\circ} \mathrm{C}$. The supernatant was collected at predetermined time points, 
being the medium renewed with $1 \mathrm{ml}$ of PBS. The amounts of SV released were determined by liquid chromatography-mass spectrometry (LC-MS). The conditions of analysis were the following:

LC-MS was performed with a mobile phase that consisted of acetonitrile $(\mathrm{ACN})+0.1 \% \mathrm{v} / \mathrm{v}$ formic acid and water $\left(\mathrm{H}_{2} \mathrm{O}\right)+$ $0.1 \% \mathrm{v} / \mathrm{v}$ formic acid, with a gradient of acetonitrile from 5 to $100 \%$ for $30 \mathrm{~min}$ and a flow rate of $0.2 \mathrm{ml} \mathrm{min}^{-1}$. Charged ions $\left([\text { simvastatin }+\mathrm{H}]^{+} \text {at } m / z=420.57 \text { and [simvastatin acid }+\mathrm{H}\right]^{+}$at $m / z=437.59$ ) were analyzed in order to determine the amount of simvastatin present in the samples.

The release of SV was fitted according to the Higuchi and Korsmeyer-Peppas mathematical kinetic models.

\section{In vitro cell culture}

rBMSCs were cultured with the membranes and their osteostimulatory potential evaluated. rBMSCs were isolated from healthy male Wistar rats with Radboudumc Animal Ethics Committee approval (RU-DEC 2014-157). Cell culture medium and all supplements were purchased from Gibco (Gibco®, Life Technologies, USA), except the fetal bovine serum (FBS; SigmaAldrich, USA). rBMSCs were isolated and cultured using a method adapted from Maniatopoulos et al. ${ }^{42}$ The tibia of male Wistar rats were extracted, and epiphyses were cut off. Cells were flushed out of the remaining diaphyses using cell culture medium consisting of $\alpha$-MEM supplemented with $10 \% \mathrm{v} / \mathrm{v}$ fetal calf serum, $50 \mathrm{mg} \mathrm{ml}^{-1}$ ascorbic acid, $10^{-8} \mathrm{M}$ dexamethasone, $50 \mathrm{mg} \mathrm{ml} \mathrm{m}^{-1}$ gentamycin and $10 \mathrm{mM}$ sodium $\beta$-glycerophosphate. The flush-out of the two tibias was cultured for one day in three $75 \mathrm{~cm}^{2}$ culture flasks in a humidified incubator $(5 \%$ $\mathrm{CO}_{2} ; 37^{\circ} \mathrm{C}$ ), after which the medium was refreshed to remove non-adherent cells. The osteogenic potential of the cells was assessed by culturing rBMSCs with an osteogenic medium for 28 days, presenting the cells mineralization capability.

A preliminary study was firstly performed to evaluate the cytotoxicity of simvastatin. Briefly, rBMSCs were cultured in a growth medium ( $\alpha$-MEM supplemented with $10 \%$ v/v FBS, 100 units per ml penicillin, and $100 \mu \mathrm{g} \mathrm{ml}^{-1}$ streptomycin) containing different concentrations of simvastatin (4200 $\mathrm{ng} \mathrm{ml}^{-1}$ or $\approx 10^{-5} \mathrm{M}, 420 \mathrm{ng} \mathrm{ml}{ }^{-1}$ or $\approx 10^{-6} \mathrm{M}, 42 \mathrm{ng} \mathrm{ml}{ }^{-1}$ or $\approx 10^{-7} \mathrm{M}$, and $4.2 \mathrm{ng} \mathrm{ml}^{-1}$ or $\approx 10^{-8} \mathrm{M}$ ) at a cell density equal to $2 \times 10^{4}$ / $\mathrm{cm}^{2}$ and incubated in a humidified incubator $\left(37^{\circ} \mathrm{C}, 5 \% \mathrm{CO}_{2}\right)$, cytotoxicity was evaluated both by visual examination with an optical microscope (DM IL LED, Leica, The Netherlands), for the cells cultured at 3 days, 14 days and 28 days, and by an AlamarBlue ${ }^{\circledR}$ viability test (Molecular Probes ${ }^{\mathrm{TM}}$, Invitrogen ${ }^{\circledR}$, Thermo Fisher Scientific, Bleiswijk, The Netherlands) after 3, 7, 14, 21 and 28 days, according to the manufacturer's instructions to obtain quantitative data on cellular activity. Viability percentages are relative to a positive control, where rBMSCs were cultured in an osteogenic medium ( $\alpha$-MEM supplemented with $10 \% \mathrm{v} / \mathrm{v}$ FBS, $50 \mathrm{mg} \mathrm{ml}^{-1}$ ascorbic acid, $10^{-8} \mathrm{M}$ dexamethasone, $10 \mathrm{mM}$ sodium $\beta$-glycerophosphate and 100 units per $\mathrm{ml}$ penicillin $+100 \mu \mathrm{g} \mathrm{ml}^{-1}$ streptomycin).

For the cell culture experiments, electrospun membranes were first disinfected with Argon plasma for 5 minutes (plasma cleaner/sterilizer PDC-001; Harrick, USA). rBMSCs were statically seeded in 24-well adherent culture plates with growth medium at a cell density equal to $2 \times 10^{4} / \mathrm{cm}^{2}$ and incubated in a humidified incubator $\left(37^{\circ} \mathrm{C}, 5 \% \mathrm{CO}_{2}\right)$, allowing the cells to adhere to the bottom of the wells. After 24 hours, membranes were placed in cell culture inserts (Thincert ${ }^{\mathrm{TM}}$, Greiner Bio-one, The Netherlands) and indirectly cultured with the cells. The different groups and conditions of culture are described in more detail in Table 1.

rBMSCs DNA quantification and ALP (alkaline phosphatase) activity measurements $(n=3)$ were performed at 3 days, 7 days, 14 days, 21 days and 28 days of co-culture as described previously. ${ }^{43}$ Briefly, cells were washed with PBS twice, collected and immersed in $1 \mathrm{ml}$ of Milli-Q water. Afterward, sonication and three freeze-thaw cycles were performed. DNA content in the supernatant was measured using a Quant-IT ${ }^{\mathrm{TM}}$ PicoGreen ${ }^{\circledR}$ dsDNA assay kit (Invitrogen; The Netherlands) according to the manufacturer's instructions. In a 96-well plate, $100 \mu \mathrm{l}$ of sample or standard were added to a working solution. The plates were incubated at room temperature in the dark for 5 minutes and fluorescence read (excitation: 485/20 nm; emission: 530/25 nm). ALP activity of the same sample was measured by a $p$-nitrophenyl phosphate (4-NP; Sigma-Aldrich, USA) colorimetric assay, according to the manufacturer's instructions. $80 \mu \mathrm{l}$ of samples or standards were added to $20 \mu \mathrm{l}$ of buffer and $100 \mu \mathrm{l}$ of the substrate. The mixture was incubated for 60 minutes at $37{ }^{\circ} \mathrm{C}$. Afterward, $100 \mu \mathrm{l}$ of $0.5 \mathrm{M} \mathrm{NaOH}$ solution was added to stop the reaction. Absorbance at $405 \mathrm{~nm}$ was read and ALP activity normalized according to the DNA content for each sample.

To observe the cellular morphology, cells were directly cultured on the membranes with the specific medium indicated in Table 1. Samples were collected at 3 days, 14 days and 28 days. A $2 \% \mathrm{v} / \mathrm{v}$ solution of glutaraldehyde (glutaraldehyde solution 25\%; Merck; Germany) was used to fixate the cells and ethanol at graded concentrations $(70 \%, 80 \%, 90 \%, 96 \%$ and $100 \% \mathrm{v} / \mathrm{v}$ ) was used for dehydration. Finally, samples were covered with tetramethylsilane (TMS; $\geq 99.0 \%$ GC; SigmaAldrich; USA) and prepared for SEM observation as referred previously.

\section{Statistical analysis}

Fiber diameter was estimated from the corresponding SEM images using Image software (National Institutes of Health, USA). Twenty different measurements were taken from 5 different regions of each electrospun membrane $(n=100)$. Data were reported as mean \pm standard deviation (SD). Statistical analysis was carried out by using one-way analysis of variance (ANOVA) combined with a Tukey's multiple comparison post-

Table 1 Groups for in vitro cell culture experiments

\begin{tabular}{ll}
\hline Group & Composition \\
\hline PLLA & Membrane + growth medium+10 mM $\beta$-GP \\
PLLA + SV & Membrane + growth medium+10 mM $\beta$-GP \\
PLLA + SV HT & Membrane + growth medium+10 mM $\beta$-GP
\end{tabular}


hoc test. Differences were considered significant at $p<0.05$. Statistical differences in crystallinity of the different samples $(n$ $=3$ ) were analyzed using an unpaired $t$-test with Welch's correction, calculated with Prism 6.01 (GraphPad Software, Inc.; USA). Data were reported as mean \pm standard deviation (SD). Differences were considered significant at $p<0.05$. DNA content and ALP activity $(n=3)$ were analyzed using Prism 6.01 and performing a two-way ANOVA significance test combined with a post-hoc Tukey's multiple comparison test. Data were reported as mean \pm standard deviation (SD). Differences were considered significant at $p<0.05$.

\section{Results}

\section{Morphological and structural evaluation of electrospun membranes containing SV}

Fig. 1 shows the morphology of the electrospun PLLA membranes with and without SV before and after thermal treatment (HT). All membranes are composed of micrometric fibers, with a small decrease of the diameter of the fibers when the drug was incorporated $(1.4 \pm 0.14 \mu \mathrm{m}$ for PLLA; $1.3 \pm 0.11$ $\mu \mathrm{m}$ for PLLA HT; $1.0 \pm 0.10 \mu \mathrm{m}$ for PLLA $+\mathrm{SV} ; 0.9 \pm 0.07 \mu \mathrm{m}$ for PLLA + SV HT). No noticeable changes in the morphology of the fibers were detected when SV was incorporated (Fig. 1c and d). Membranes subjected to the thermal treatment (PLLA HT and PLLA + SV HT) present a similar morphology when compared with their non-thermal treated counterparts (PLLA and PLLA + SV), although some deformation was visible of the fibers (Fig. 1b-red square and Fig. 1d-red square).

The chemical profile of the membranes was analyzed by ATRIR (Fig. 2). The spectra were similar for all membranes, showing the characteristic peaks of PLLA highlighted with red lines: $1751 \mathrm{~cm}^{-1}$ (carbonyl group); 1000-1450 $\mathrm{cm}^{-1}$ (ether groups) and $870 \mathrm{~cm}^{-1}$ (alkyl groups). ${ }^{32,44,45}$ No characteristic peaks related to the presence of SV were detected, either before or after thermal treatment. Further, thermal treatment did not affect the chemical composition of the membranes, with no significant changes in the polymeric backbone.

Fig. 3 presents the XRD profiles for the different membranes. Samples without thermal treatment showed no sharp peaks on the XRD spectra. Membranes with thermal treatment showed a peak at approximately $2 \theta=16^{\circ}$ (red arrows, Fig. 3), representing the characteristic peak of the crystal structure of the $\alpha$ form of PLLA. ${ }^{46}$

Fig. 4 shows the crystallinity percentage of the different electrospun membranes. As expected, crystallinity increased after the membranes were subjected to thermal treatment, shown by XRD ( $42 \pm 7.8 \%$ vs. $58 \pm 4.4 \%$ for PLLA without SV and $38 \pm 3.0 \%$ vs. $62 \pm 1.1 \%$ for PLLA with SV). On the other hand, the incorporation of SV did not affect the crystallinity of PLLA. The representative DSC spectra of each group are shown in Fig. $\mathrm{S} 1 \dagger$ and the correlated thermal properties are summarized in Table S1. $\dagger$ The glass transition temperature $\left(T_{\mathrm{g}}\right)$ decreased when SV was incorporated $\left(54 \pm 1.1{ }^{\circ} \mathrm{C} v s .46 \pm 0.1{ }^{\circ} \mathrm{C}\right.$ for PLLA and PLLA $+\mathrm{SV}$, respectively). $T_{\mathrm{g}}$ of membranes subjected to thermal treatment was not detectable considering the total heat flow (Fig. S1†). However, when examining the data obtained with the reversing heat flow, the $T_{\mathrm{g}}$ for these membranes was determined (Fig. $\mathrm{S} 2 \dagger$ ). A substantial increase of the $T_{\mathrm{g}}$ values due to thermal treatment was detected $\left(73 \pm 0.3^{\circ} \mathrm{C}\right.$ and $73 \pm 1.0^{\circ} \mathrm{C}$ for PLLA HT and PLLA $+\mathrm{SV}$ HT respectively). The melting temperature $\left(T_{\mathrm{m}}\right)$ was not affected by the incorporation of SV or by the thermal treatment.

\section{Drug release from PLLA membranes}

The release profiles of the membranes containing SV are presented in Fig. 5. No initial burst release of SV was detected for both membranes. A higher SV release from PLLA without
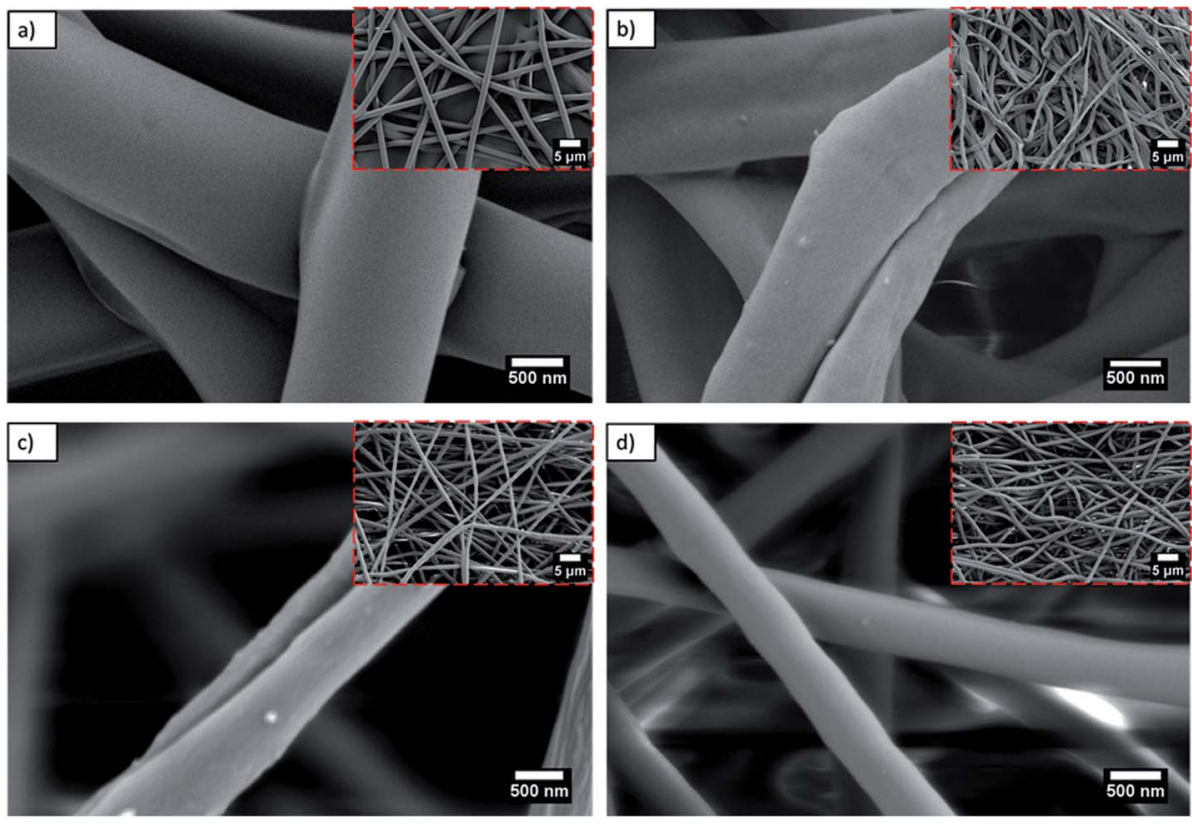

Fig. 1 SEM images of electrospun fibers 5000× (red squares) and $50000 \times$. (a) PLLA (b) PLLA HT (c) PLLA + SV (d) PLLA + SV HT. 


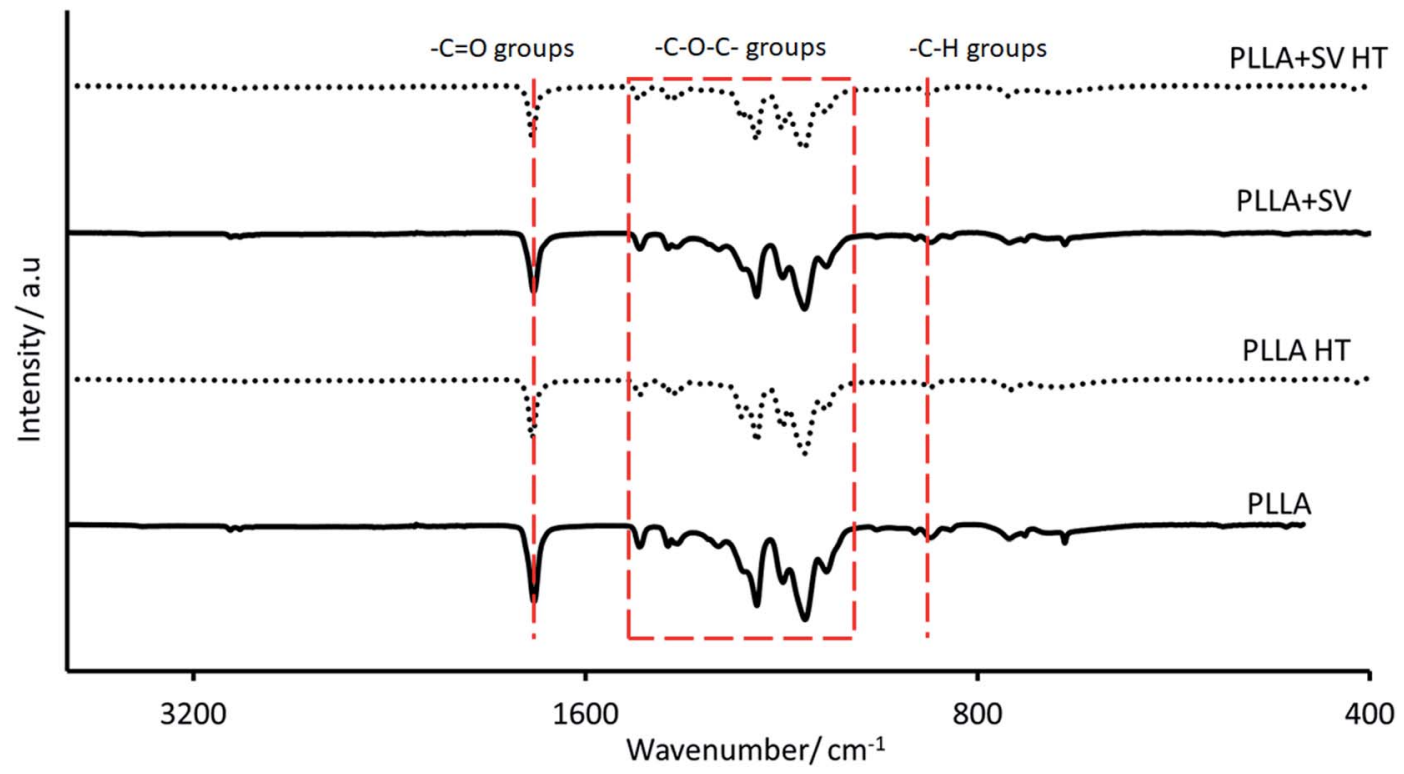

Fig. 2 ATR-IR spectra of electrospun membranes. Red lines represent the main peaks belonging to PLLA.

thermal treatment was detected. SV released at a fast speed within the first 5 days and continued to release at a constant slow speed up to 28 days, except that PLLA + SV showed a sudden increase in the amount of SV released at week 4 . Not all the incorporated SV was released from PLLA membranes after 4 weeks: $11 \pm 1.9 \%$ SV released for PLLA + SV and $3 \pm 0.8 \%$ for PLLA + SV HT.

SV release data were fitted according to two well-established drug release models: the Higuchi model and the KorsmeyerPeppas model. As shown in Fig. S3, $\dagger$ SV release correlated highly with both models, although the Korsmeyer-Peppas model presented a higher correlation for both membranes $\left(R^{2}=0.98\right.$ vs. $R^{2}=0.97$ for Higuchi model). The Korsmeyer-Peppas model fitting showed different release exponents $(n): n=0.56$ for PLLA + SV and $n=0.47$ for PLLA + SV HT.

\section{In vitro study with rBMSCs}

rBMSCs were cultured in a proliferative medium containing simvastatin at different concentrations for 28 days, and cytotoxicity was evaluated using light microscopy and AlamarBlue assays (Fig. S4 and S5†). Cellular proliferation occurred mainly

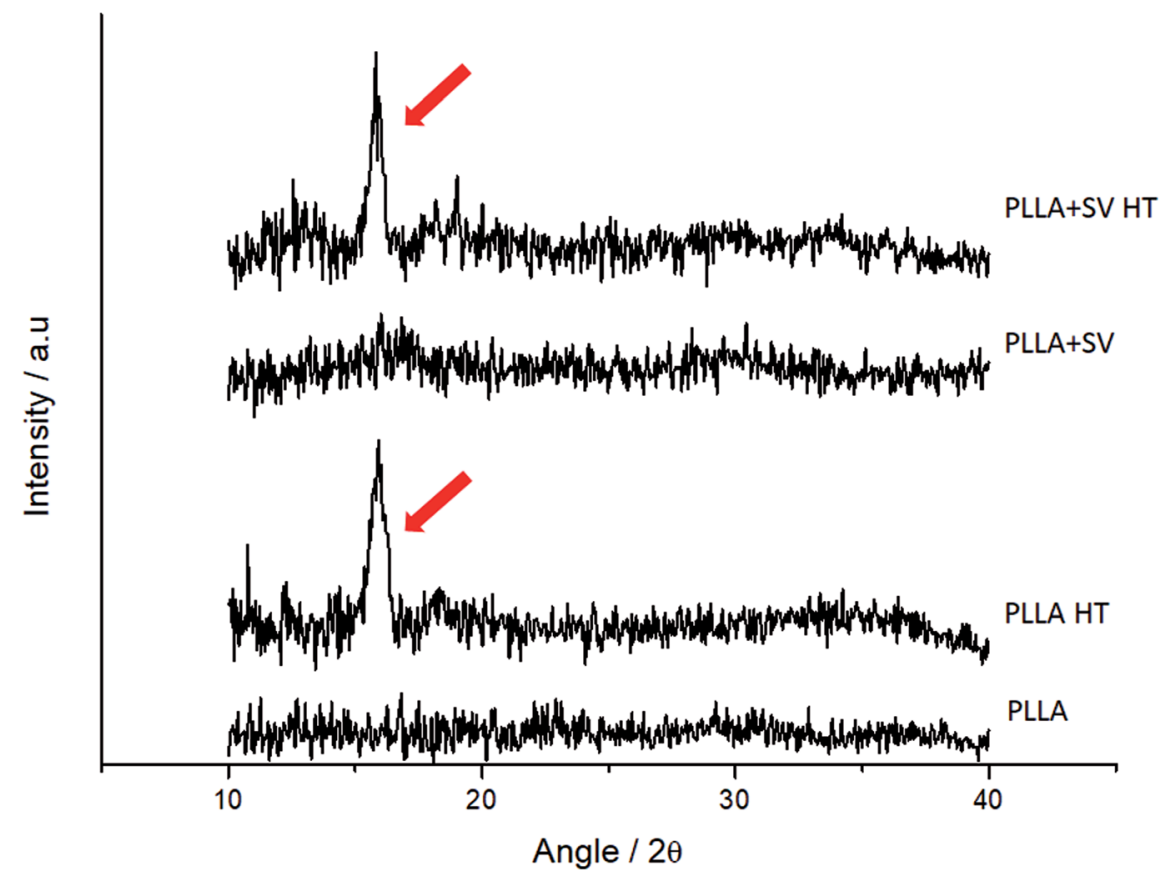

Fig. 3 XRD spectra of electrospun membranes. Red arrows indicate the peak of the $\alpha$ form of PLLA. 


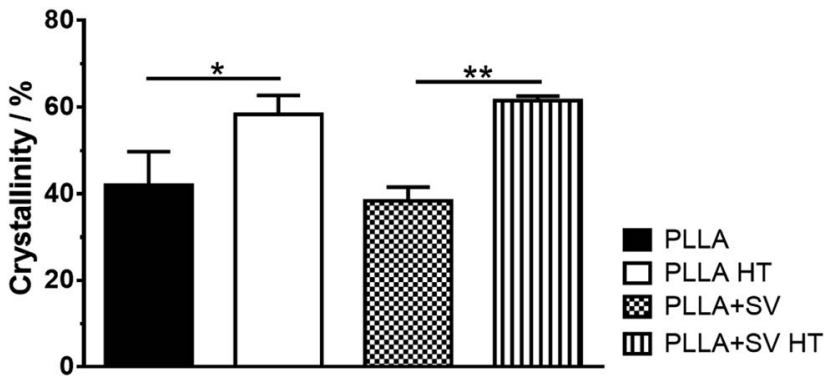

Fig. 4 Crystallinity of electrospun membranes. $*=p<0.05$; $* *=p<0.01$

for the cells cultured with simvastatin concentrations of $42 \mathrm{ng}$ $\mathrm{ml}^{-1}\left(10^{-7} \mathrm{M}\right)$ and $4.2 \mathrm{ng} \mathrm{ml}{ }^{-1}\left(10^{-8} \mathrm{M}\right)$. Simvastatin concentrations above $420 \mathrm{ng} \mathrm{ml}^{-1}\left(\approx 10^{-6} \mathrm{M}\right)$ resulted in substantial cytotoxicity, leading to cellular death (Fig. S4†) and low viability percentages (Fig. S5†).

rBMSCs were directly seeded on the different electrospun membranes with the purpose to analyze the cellular morphology and response. Fig. 6 shows the images obtained by SEM after 3, 14 and 28 days of culture. After 3 days of culture, cells showed adherence to the membranes. At 14 days of culture, the number of cells increased significantly, covering almost the entire surface of the membranes. At 28 days, the membranes were completely covered by the cells and a thick cell layer on top of the membranes was observed.

Fig. 7 shows the DNA amount and ALP-activity obtained upon indirect in vitro culture experiments. An increase in the DNA amount with time was observed for all groups. At individual time points, no significant differences between groups were observed. PLLA + SV HT membranes evoked an increase in ALP-activity up to 7 days followed by a decrease. Further, PLLA + SV HT membranes induced the highest ALP-activity levels among all groups (Fig. 7b).

\section{Discussion}

We here aimed to incorporate an osteostimulatory drug, simvastatin (SV), in a PLLA electrospun membrane and prolong its release, with the ultimate objective of creating a membrane with suitable biological properties for GBR applications. SV was chosen not only due to its well documented promotion of in vitro osteogenic differentiation ${ }^{47,48}$ and bone regeneration, both in in vivo ${ }^{\mathbf{4 9 5}}$ and in clinical studies, ${ }^{51}$ but also because it possesses a high thermal stability $\left(T_{\mathrm{m}}\right.$ of $\left.139{ }^{\circ} \mathrm{C}\right)$, and good solubility in organic solvents. ${ }^{\mathbf{5 2 , 5 3}}$ The effect of PLLA crystallinity on the release of SV was investigated. In order to increase PLLA crystallinity, membranes were treated at $80{ }^{\circ} \mathrm{C}$ for 16 hours. SV release kinetics showed that variations in PLLA crystallinity can be used to control SV release. In vitro cell culture assays demonstrated a continuous proliferation of cells over time for all compositions, with a significant increase in ALP-activity levels of osteogenic cells by membranes containing SV.

PLLA membranes containing SV were produced by electrospinning. Morphological evaluation of the membranes showed the formation of micrometric electrospun fibers with a smooth surface and a decrease of the diameter of the fibers when SV was incorporated. The decrease in fiber diameter is probably related to an increase in the conductivity of the polymeric solution for the compositions containing SV, leading to a decrease in fiber diameter during electrospinning. ${ }^{54}$ Thermal treatment did not affect the surface morphology or the diameter of the fibers, although limited fiber deformation could be noticed.

Similar ATR-IR spectra were obtained for all the membranes due to the overlap of characteristic infrared bands of SV with those of PLLA. ${ }^{52,55}$ Further, the similar spectra of membranes with or without thermal treatment indicate that the thermal treatment did not change the chemical structure of the electrospun membranes.

XRD and DSC results confirmed that the thermal treatment used in this study increased the crystallinity of PLLA membranes. It has been shown previously that an increase in polymer crystallinity due to thermal exposure leads to a slower diffusion of the drugs because the crystalline domains function as a physical barrier and reduce polymeric degradation. ${ }^{56,57}$ The results from our study corroborate these data. The amount of SV released from PLLA + SV membranes was approximately 2 times higher than the membranes subjected to the thermal treatment (PLLA + SV HT), demonstrating the primary role of crystallinity in controlling the release of SV. Release data of both membranes were fitted according to two kinetic models (i.e. the Higuchi model and the Korsmeyer-Peppas model) in order to understand the drug release mechanism(s)..$^{58,59}$ The Higuchi
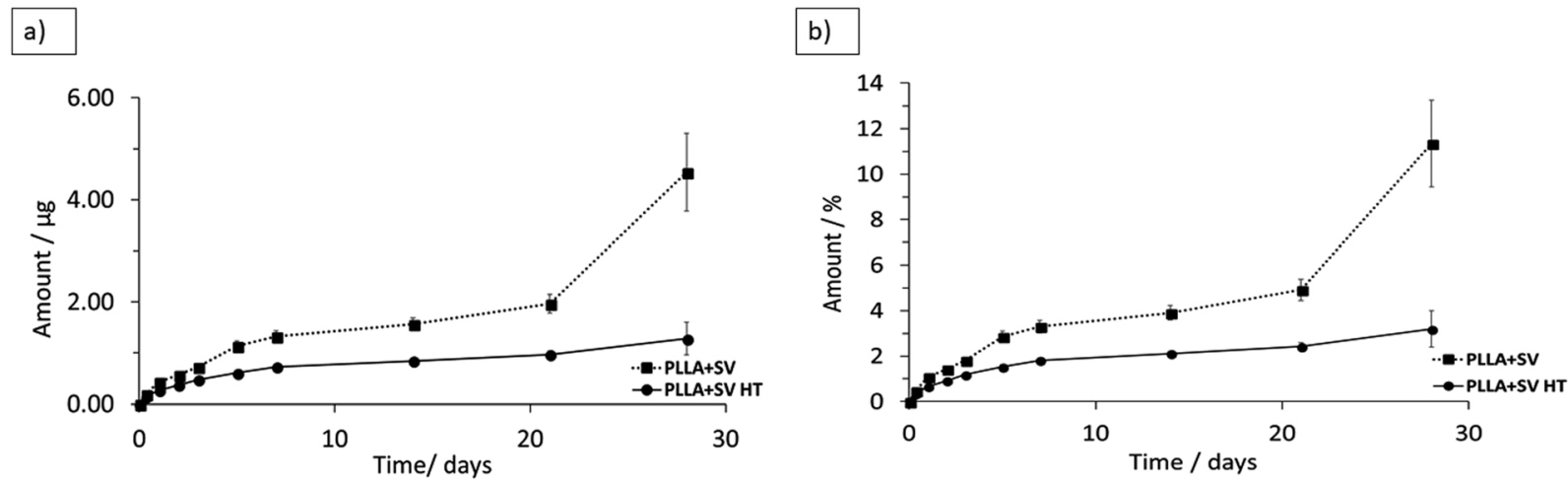

Fig. 5 Cumulative release of simvastatin in PBS at $37^{\circ} \mathrm{C}$ (a) cumulative mass (b) cumulative percentage. 

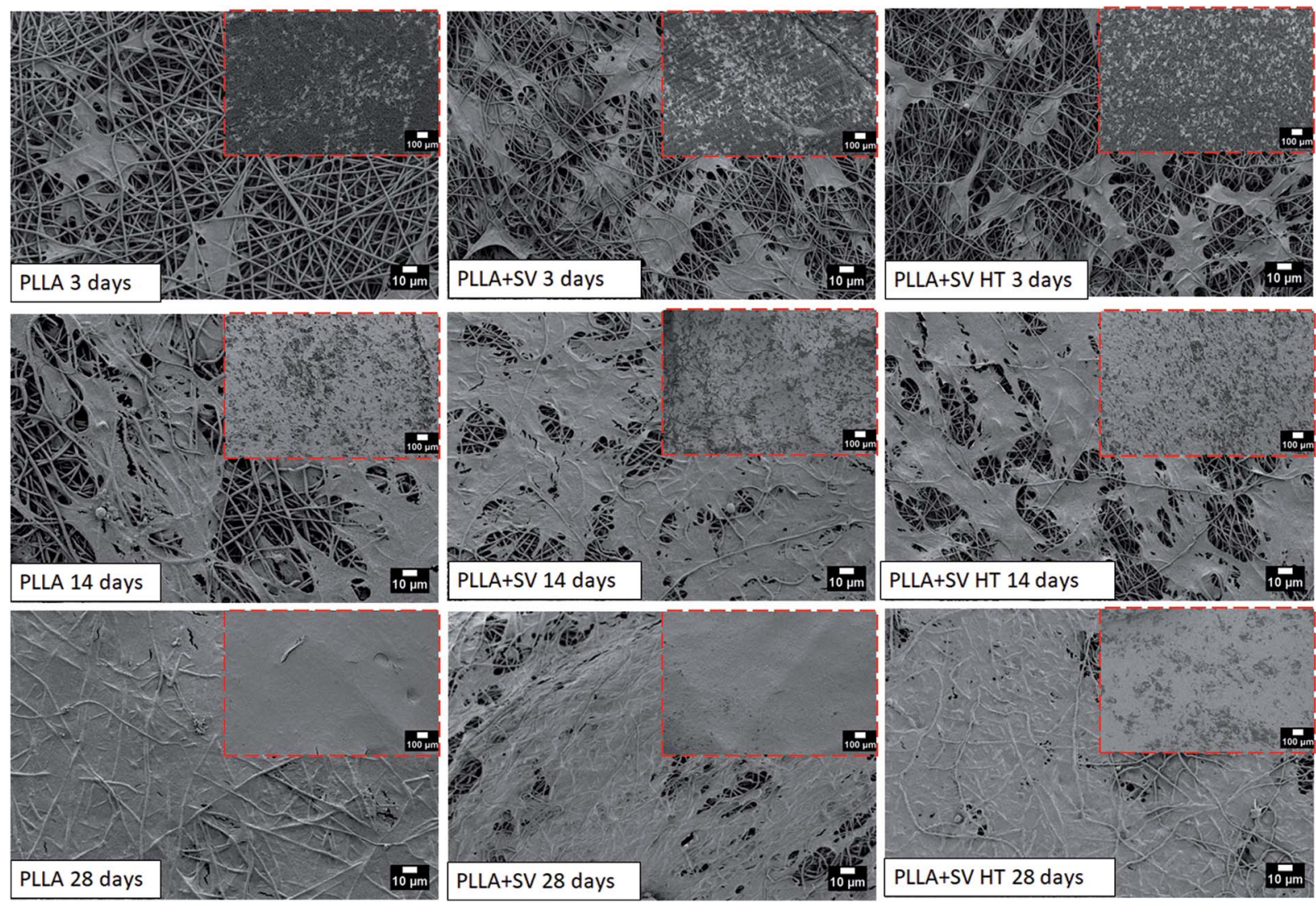

Fig. 6 SEM images of rBMSCs cultured on the membranes at $150 \times$ (red squares) and $1000 \times$

kinetic model is an ideal model, which considers the release of a drug from a homogeneous solid matrix, where the diffusion of the drug is constant and phenomena like polymer swelling and dissolution are negligible. The Korsmeyer-Peppas kinetic model is more complex and different release mechanisms are considered. According to the Korsmeyer-Peppas model, for cylindrical structures an $n \leq 0.45$ corresponds to fickian-based diffusion, an $0.45<n<0.89$ to non-fickian (anomalous) diffusion, an $n=0.89$ to case II (relaxational) transport, and $n>0.89$ to super case II transport, the two last mechanisms being related to polymer swelling and erosion. For both models, the correlation coefficient was high, although slightly higher for the
Korsmeyer-Peppas kinetic model. The Korsmeyer-Peppas model was considered as more accurate to explain the mechanism(s) related to the in vitro release of SV. Considering that ideally the electrospun fibers possess a cylindrical geometry, the determined $n$ values demonstrate that PLLA + SV membranes exert an anomalous non-fickian diffusion of SV to the medium $(n>0.45)$, likely due to fiber erosion phenomena. PLLA + SV HT membranes, on the other hand, present an $n$ value very close to 0.45 , suggesting a diffusion of SV that follows the Fick's law. ${ }^{60}$

rBMSCs were cultured with pure and SV-loaded PLLA membranes with or without thermal treatment (PLLA, PLLA + SV and PLLA + SV HT). The mass of the membranes utilized in a)

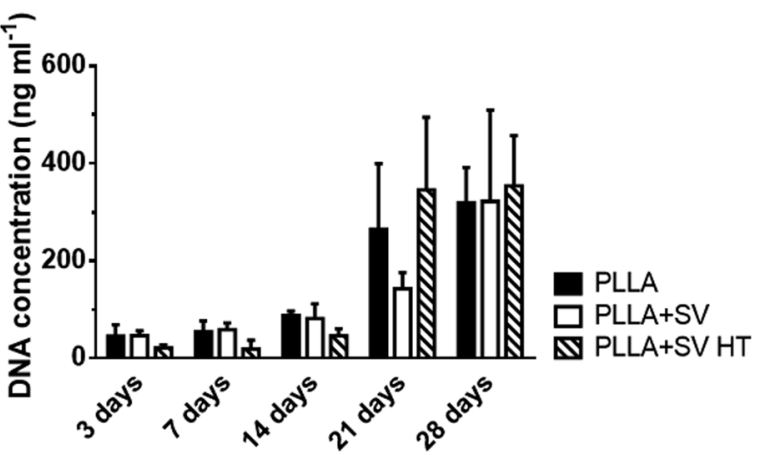

b)

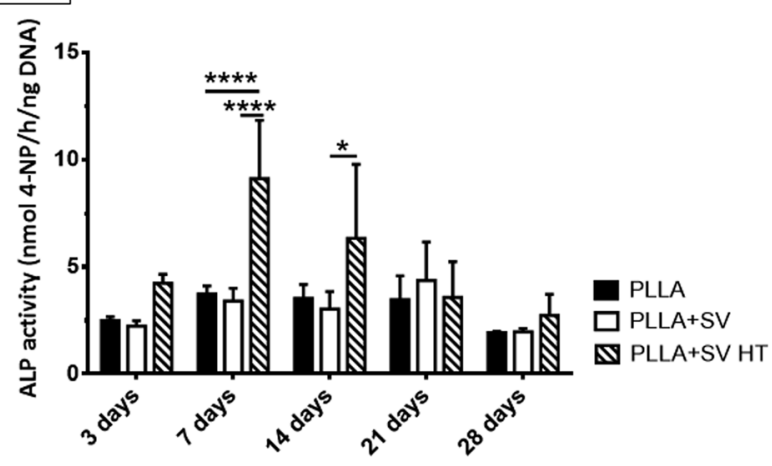

Fig. 7 In vitro culture of rBMSCs. (a) DNA concentration (b) ALP activity. ${ }^{*}=p<0.05 ; * * * *=p<0.0001$. 
culture was calculated based on the SV release profile so that the concentration of SV in the medium was within the reported range capable of inducing an osteogenic response. ${ }^{\mathbf{2 0 , 2 5 , 6 1 , 6 2}}$ Should be mentioned that the potential cytotoxicity of simvastatin was also considered. Viability tests, where rBMSCs were cultured in medium containing different concentrations of simvastatin, were performed on an early phase of this study (Fig. S4 and S5 $\dagger$ ) and the following assays were performed taking into account the viability results obtained.

The purpose of these experiments was to evaluate if (i) the membranes could induce an osteogenic response from the rBMSCs, and (ii) the differences in the amount of drug released from the membranes with or without thermal treatment would affect osteogenic differentiation. Morphological observations indicated that rBMSCs adhered and proliferated on all membranes over time. The results of DNA assay were consistent with this finding. The DNA results further demonstrated that the tested membranes did not evoke a cytotoxic effect. Moreover, SV incorporation followed by thermal treatment induced an increased osteogenic response from rBMSCs, demonstrated by a significantly increased ALP-activity for the cells cultured on such membranes (PLLA + SV HT). A suppression of an initial burst-release, combined with the significantly lower amounts of SV present in the medium, due to its more sustained release from these membranes, appeared to be beneficial for the osteogenic differentiation of pre-osteoblastic cells. ${ }^{25,60}$ In this study a non-osteogenic medium supplemented with $\beta$-glycerophosphate was used to solely evaluate the effect of SV on the osteogenic differentiation of rBMSCs, without the influence of known osteoinductive compounds as ascorbic acid or dexamethasone. Also, we focused on analyzing the ALP-activity levels, since SV is known to have a major effect in the expression of this protein and a significant impact on the early stages of the osteogenic differentiation of osteoblastic cells. ${ }^{50}$ Further studies should be performed in order to evaluate the effect of the systems developed on the mineralization capability of rBMSCs.

\section{Conclusion}

We developed electrospun PLLA membranes with enhanced osteostimulatory properties by incorporating simvastatin (SV) and modified the SV release profile by varying the PLLA crystallinity. Thermal treatment successfully increased the PLLA crystallinity for all compositions without significantly changing the chemical properties and morphology of the electrospun fibers. A markedly more sustained release of SV was observed upon the thermal treatment to increase the PLLA crystallinity. In vitro culture of PLLA membranes with rBMSCs demonstrated that the membranes are cytocompatible, with an increase in cell proliferation over time. PLLA + SV HT induced an osteogenic response from rBMSCs, with significantly increased ALP-activity levels demonstrating both a preservation of biological activity of SV and an appropriate SV release profile. Thermal treatment to increase PLLA crystallinity in electrospun membranes can be used to control SV release and render these membranes biologically active.

\section{Conflicts of interest}

There are no conflicts to declare.

\section{Acknowledgements}

The authors acknowledge the Dutch government to the Netherlands Institute for Regenerative Medicine (NIRM, grant no. FES0908) for the financial support for this research.

\section{References}

1 I. Elgali, O. Omar, C. Dahlin and P. Thomsen, Eur. J. Oral Sci., 2017, 125, 315-337.

2 M. Retzepi and N. Donos, Clin. Oral Implants Res., 2010, 21, 567-576.

3 P. Gentile, V. Chiono, C. Tonda-Turo, A. M. Ferreira and G. Ciardelli, Biotechnol. J., 2011, 6, 1187-1197.

4 D. H. Reneker and A. L. Yarin, Polymer, 2008, 49, 2387-2425.

5 F. Yang, S. K. Both, X. Yang, X. F. Walboomers and J. A. Jansen, Acta Biomater., 2009, 5, 3295-3304.

6 H. Zhang, J. Wang, K. Wang and L. Xu, Mater. Lett., 2018, 212, 118-121.

7 X. Sun, C. Xu, G. Wu, Q. Ye and C. Wang, Polymers, 2017, 9, 189.

8 J. L. Wang, L. N. Wang, Z. Y. Zhou, H. J. Lai, P. Xu, L. Liao and J. C. Wei, Polymers, 2016, 8, 115.

9 D. Huang, L. Niu, J. Li, J. Du, Y. Wei, Y. Hu, X. Lian, W. Chen and K. Wang, J. Mech. Behav. Biomed. Mater., 2018, 81, 195201.

10 H. Zhang, G. Gruner and Y. Zhao, J. Mater. Chem. B, 2013, 1, 2542-2567.

11 U. S. Shin, J. S. Park, J. S. Oh and E. J. Lee, Macromol. Res., 2017, 25, 1057-1062.

12 Y. J. Lee, J. H. Lee, H. J. Cho, H. K. Kim, T. R. Yoon and H. Shin, Biomaterials, 2013, 34, 5059-5069.

13 H. Nie, B. W. Soh, Y. C. Fu and C. H. Wang, Biotechnol. Bioeng., 2008, 99, 223-234.

14 T. Jiang, E. J. Carbone, K. W. H. Lo and C. T. Laurencin, Prog. Polym. Sci., 2015, 46, 1-24.

15 W. Ji, Y. Sun, F. Yang, J. J. J. P. van den Beucken, M. Fan, Z. Chen and J. A. Jansen, Pharm. Res., 2011, 28, 1259-1272.

16 E. J. Carbone, T. Jiang, C. Nelson, N. Henry and K. W. H. Lo, Nanomedicine, 2014, 10, 1691-1699.

17 H. A. Fathi, A. Allam, M. Elsabahy, G. Fetih and M. El-Badry, Colloids Surf., B, 2018, 162, 236-245.

18 M. Patel and C. Kothari, Trends Anal. Chem., 2017, 86, 206221.

19 M. Zhang, Y. Q. Bian, H. M. Tao, X. F. Yang and W. D. Mu, Eur. Rev. Med. Pharmacol. Sci., 2018, 22, 2896-2905.

20 W.-L. Yu, T.-W. Sun, C. Qi, H.-K. Zhao, Z.-Y. Ding, Z.-W. Zhang, B.-B. Sun, J. Shen, F. Chen, Y.-J. Zhu, D.-Y. Chen and Y.-H. He, Sci. Rep., 2017, 7, 44129.

21 J. Chou, T. Ito, D. Bishop, M. Otsuka, B. Ben-Nissan and B. Milthorpe, PLoS One, 2013, 8, e54676.

22 M. Lai, Z. Y. Jin, X. Y. Yang, H. Y. Wang and K. Xu, Appl. Surf. Sci., 2017, 396, 1741-1751. 
23 F. Yang, S. F. Zhao, F. Zhang, F. M. He and G. L. Yang, Oral Surg. Oral Med. Oral Pathol. Oral Radiol. Endod., 2011, 111, 551-556.

24 P. C. Chang, A. S. Dovban, L. P. Lim, L. Y. Chong, M. Y. Kuo and C. H. Wang, Biomaterials, 2013, 34, 9990-9997.

25 X. Liu, X. Li, L. Zhou, S. Li, J. Sun, Z. Wang, Y. Gao, Y. Jiang, H. Lu, Q. Wang and J. Dai, Colloids Surf., B, 2013, 102, 420427.

26 J. B. Lee, J. E. Kim, D. A. Balikov, M. S. Bae, D. N. Heo, D. Lee, H. J. Rim, D. W. Lee, H. J. Sung and I. K. Kwon, Macromol. Biosci., 2016, 16, 1027-1038.

27 K. M. Whang, E. Grageda, A. Khan, J. McDonald, M. Lawton and N. Satsangi, J. Biomed. Mater. Res., Part A, 2005, 74a, 247253.

28 M. Kouhi, M. Morshed, J. Varshosaz and M. H. Fathi, Chem. Eng. J., 2013, 228, 1057-1065.

29 M. Samiei, M. Aghazadeh, E. Alizadeh, N. Aslaminabadi, S. Davaran, S. Shirazi, F. Ashrafi and R. Salehi, Adv. Pharm. Bull., 2016, 6, 353-365.

30 J. Liu and D. G. Kerns, Open Dent. J., 2014, 8, 56-65.

$31 \mathrm{H}$. Tsuji and Y. Ikada, Polymer, 1995, 36, 2709-2716.

32 J. Zhang, Y. Duan, A. J. Domb and Y. Ozaki, Macromolecules, 2010, 43, 4240-4246.

33 T. Zhang, J. Hu, Y. Duan, F. Pi and J. Zhang, J. Phys. Chem. B, 2011, 115, 13835-13841.

34 S. D. Yoon, Y. S. Kwon and K. S. Lee, Int. Neurourol. J., 2017, 21, S48-S54.

35 K. A. Blackwood, R. McKean, I. Canton, C. O. Freeman, K. L. Franklin, D. Cole, I. Brook, P. Farthing, S. Rimmer, J. W. Haycock, A. J. Ryan and S. MacNeil, Biomaterials, 2008, 29, 3091-3104.

36 I. Schlegel, R. Munoz-Espi, P. Renz, I. Lieberwirth, G. Floudas, Y. Suzuki, D. Crespy and K. Landfester, Macromolecules, 2017, 50, 4725-4732.

37 A. J. Ro, R. Falotico and V. Davé, J. Biomed. Mater. Res., Part B, 2011, 97B, 30-39.

38 H. Lian and Z. Meng, Mater. Sci. Eng., C, 2017, 74, 117-123.

39 S. Sene, J. McLane, N. Schaub, S. Begu, P. H. Mutin, L. Ligon, R. J. Gilbert and D. Laurencin, J. Mater. Chem. B, 2016, 4, 257-272.

40 M. Miyajima, A. Koshika, J. i. Okada, M. Ikeda and K. Nishimura, J. Controlled Release, 1997, 49, 207-215.

41 J. F. Turner, A. Riga, A. O'Connor, J. Zhang and J. Collis, J. Therm. Anal. Calorim., 2004, 75, 257-268.
42 C. Maniatopoulos, J. Sodek and A. H. Melcher, Cell Tissue Res., 1988, 254, 317-330.

43 A. G. B. Castro, M. Diba, M. Kersten, J. A. Jansen, J. J. J. P. van den Beucken and F. Yang, Mater. Sci. Eng., C, 2018, 85, 154161.

44 J. P. Chen and C. H. Su, Acta Biomater., 2011, 7, 234-243.

45 B. Liu, F. Xu, M. Y. Guo, S. F. Chen, J. Wang and B. Zhang, Surf. Coat. Technol., 2013, 228, S568-S573.

46 O. Monticelli, S. Bocchini, L. Gardella, D. Cavallo, P. Cebe and G. Germelli, Eur. Polym. J., 2013, 49, 2572-2583.

47 H. Pullisaar, H. Tiainen, M. A. Landin, S. P. Lyngstadaas, H. J. Haugen, J. E. Reseland and E. Ostrup, J. Tissue Eng., 2013, 4, 2041731413515670.

48 M. Lopez-Alvarez, V. Lopez-Puente, C. Rodriguez-Valencia, P. C. Angelome, L. M. Liz-Marzan, J. Serra, I. PastorizaSantos and P. Gonzalez, Biomed. Mater., 2018, 13, 025017.

49 L. Dai, M. Xu, H. Wu, L. Xue, D. Yuan, Y. Wang, Z. Shen, H. Zhao and M. Hu, J. Bone Miner. Metab., 2016, 34, 23-32.

50 J. Hao, J. Chou, S. Kuroda, M. Otsuka, S. Kasugai and N. P. Lang, Clin. Oral Implants Res., 2018, 29, 227-234.

51 S. Degala and N. A. Bathija, J. Oral Maxillofac. Surg., DOI: 10.1016/j.joms.2018.04.035, in press.

52 A. A. Ambike, K. R. Mahadik and A. Paradkar, Drug Dev. Ind. Pharm., 2005, 31, 895-899.

53 G. Murtaza, Acta Pol. Pharm., 2012, 69, 581-590.

54 K. Kim, Y. K. Luu, C. Chang, D. F. Fang, B. S. Hsiao, B. Chu and M. Hadjiargyrou, J. Controlled Release, 2004, 98, 47-56.

55 M. S. Bhatia, S. D. Jadhav, N. M. Bhatia, P. B. Choudhari and K. B. Ingale, Sci. Pharm., 2011, 79, 601-614.

56 Z. Zhou, Q. Yi, L. Liu, X. Liu and Q. Liu, J. Macromol. Sci., Part B: Phys., 2009, 48, 309-317.

57 M. Nakagawa, F. Teraoka and T. Sohmura, J. Appl. Polym. Sci., 2011, 119, 2031-2038.

58 S. Dash, P. N. Murthy, L. Nath and P. Chowdhury, Acta Pol. Pharm., 2010, 67, 217-223.

59 P. Costa, J. Manuel and S. Lobo, Eur. J. Pharm. Sci., 2001, 13, 123-133.

60 T. Terukina, H. Saito, Y. Tomita, Y. Hattori and M. Otsuka, J. Drug Delivery Sci. Technol., 2017, 37, 74-80.

61 P.-Y. Chen, J.-S. Sun, Y.-H. Tsuang, M.-H. Chen, P.-W. Weng and F.-H. Lin, Nutr. Res., 2010, 30, 191-199.

62 M. Terauchi, T. Inada, A. Tonegawa, A. Tamura, S. Yamaguchi, K. Harada and N. Yui, Int. J. Biol. Macromol., 2016, 93, 1492-1498. 